Optimal recharging framework and simulation for electric vehicle fleet Peer-reviewed author version

USMAN, Muhammad; KNAPEN, Luk; YASAR, Ansar; BELLEMANS, Tom; JANSSENS, Davy \& WETS, Geert (2020) Optimal recharging framework and simulation for electric vehicle fleet. In: Future generation computer systems, 107, p. 745-757..

DOI: $10.1016 /$ j.future.2017.04.037

Handle: http://hdl.handle.net/1942/24090 


\title{
Optimal recharging framework and simulation for electric vehicle fleet
}

\author{
Muhammad Usmanı, Luk Knapen, Ansar-Ul-Haque Yasar, Tom Bellemans, Davy Janssens, Geert Wets
}

Transportation Research Institute (IMOB),University Hasselt, Belgium

\begin{abstract}
Electrical vehicles are considered sustainable transport alternatives as compared to conventional combustion engine vehicles due to their lower energy consumption and less pollutants production. The increased penetration of electric vehicles in the market depends upon technology to overcome the driving range barrier. That can be achieved by significantly planning the use of available charging stations. In this paper, a planning simulation model is presented which evaluates the feasibility of electric vehicles driving range when recharging is considered at home, at work or at quick charging stations in Flanders, Belgium. The proposed procedure plans a charging strategy for each electric vehicle so that entire scheduled tours of the individual can be executed successfully. The simulation starts by activating an agent for each electric vehicle that takes the daily schedule of the driver, registered charging requests at each charging station, and devises a charging strategy that may or may not require a detour to a charging station. Detouring to a charging station causes the time loss that result in the utility drop due to decreased participation in the planned activities. The charging station that leads to the minimum detour travel time, waiting time and recharging time is selected to minimize the time loss. The simulation uses a realistic travel demand predicted by an activity-based model. The results show the percentage of the population that can drive electric vehicle with charging only at home and/or work location; it also shows those who need a stop for recharging at a charging station. The results indicate the use of all charging stations over the day and also the waiting time as function of charging points at each charging station.
\end{abstract}

Keywords: Quick charging station, optimal charging, electric vehicle, time loss, simulation model.

\section{Nomenclature}

EV: Electric vehicle

PHEV: Plug-in hybrid electric vehicle

ICEV: Internal combustion engine vehicle

QCS: Quick charging station

SOC: State-of-charge in battery

HH tour: Home-to-Home tour

DCD: Deepest charging depletion threshold in battery.

DER: Distributed energy resources

\section{Introduction}

Due to lower energy consumption, energy costs and less pollutants production, recently the electrical vehicles have become a popular green transportation means[1]. Electric vehicles are sustainable transport alternatives in contrast to conventional combustion engine vehicles. EVs use one or more electric motors to partially or entirely replace the internal combustion engine and are referred as electric vehicle (EV). An electric vehicle may be powered through a collector system: by electricity from off-vehicle sources, or may be self-contained with a battery or generator to convert fuel to electricity. EVs include road and rail vehicles, surface and underwater vessels, electric aircraft and electric spacecraft. This paper focuses on BEV (battery only EV) cars.

1 Corresponding author. Tel.: +32-11-269 140; fax: +32-11- 269199.

E-mail address: muhammad.usman@uhasselt.be 
Driving range is one of the challenges to meet the expected penetration of electric vehicles in the market[2]. The distance an electric vehicle can travel with full battery is known as the driving range. The range of electric vehicles available in market varies from 60 to more than 380 miles[3]. Temperature and driving style significantly affect the distance an electric vehicle can travel with a single recharge. The recharging time of an electric vehicle depends upon the power outlet rating, the battery capacity and the initial state of charge (SOC) of the battery. Recharging the EV depends upon the battery capacity and charging infrastructure. Using regular domestic power connection, charging the battery at home takes around 6 hours when the state of the charge in the battery is at deepest charging depletion (DCD) level. In a number of cases, home wiring can be upgraded to support chargers that can deliver more electric current, lowering the time required to charge the battery. In addition to charging at home, an infrastructure of commercial quick charging stations is being deployed during recent years. In order to stopover at charging station, the electric vehicle user needs to devote some time out of the planned schedule which results in time loss [4]. To minimize the time loss for charging, the electric vehicle driver must select an optimal charging station.

To find an optimal charging station, the driver needs to know and compare the registered charging load at each station. Without an automated system, it is difficult to find an optimal charging station[5]. In this paper an automated framework is proposed that evaluates the feasibility of electric vehicles driving range when recharging at home, work or at a charging station is considered. A simulation model is mapped from the proposed framework for evaluation and testing. Representing the real systems via simulation allows exploring system behavior in an articulated way that is often either not possible or too risky in the real world[6]. The use of modeling and simulation within engineering field is important and well recognized. It belongs to the tool set of all application areas and has been included in the body of knowledge. It has been applied to a broad range of topics in transportation sciences including simulation of vehicles or pedestrian flow, car following and lane changing models, route choice modeling, and traffic simulation [7]. This is the key ingredient that drives most decisions in transportation planning and traffic operations.

The simulation model that is described in this paper calculates charging requirements of EVs depending upon travel demand and further plans a charging strategy. It starts by activating an agent for each electric vehicle which takes the daily schedule of the driver; it knows the registered charging requests at each charging station and devises a charging strategy which may or may not require a detour to a charging station. Detouring to a charging station causes the time loss which results in the utility drop due to decreased participation in the planned activities. An optimal charging station which has the minimum detour travel time, waiting time and recharging time is selected to minimize the time loss. After selecting the optimal charging station, the electric vehicle agent updates the driver's schedule by inserting a charging activity. EV agent interacts with charging station administrator to register the planned charging activity. The booked charging requests at all charging stations are administrated by an independent service. Another service which is named "power consumption tracker" in this paper, is used to track the power consumption over the day for each charging station. To evaluate the proposed framework, the population of Flanders region is simulated using the travel demand for a given day.

The paper is organized as follows: Section 2 reviews the related literature. In the first part of Section 3, we present a conceptual framework and design details. In the second part, the implementation and technical details of the simulation model are presented. Simulation experiments, required data inputs and results are reported in Section 4. Finally, conclusion and future work is presented in section 5.

\section{Literature Review}

The review focuses on the literature regarding recharging scheduling problems.

Ma et al. [8] presented a scheduling strategy through simulation studies on workday and weekend scenarios that is realized as a moving window optimization scheme. This strategy optimizes the charging costs of an EV fleet with consideration of grid constraints. Authors claim that the presented strategy can optimally plan EV charging schedules with consideration of online information and uncertainties. Cardoso et al. [9] investigated optimal sizing and scheduling of distributed energy resource (DER) capacity at a given site, considering the potential effect of electric vehicles and uncertainty in driving schedules. A novel EV fleet aggregator model was introduced and 
stochastic formulation was added to DER-CAM, a widely used deterministic model in DER sizing and scheduling problems. Alonso et al. [10] presented an optimization algorithm to coordinate the charging of EVs that was developed and implemented using a genetic algorithm which results in an optimal schedule for charging EV batteries throughout the day. The realistic behavior of drivers was used to obtain the mobility patterns and parking availability aspect of EVs to improve the charging and discharging process. Venkatesh and Guan [11] proposed a globally optimal scheduling scheme and a locally optimal scheduling scheme for EV charging and discharging. The globally optimal solution provides the globally minimal total cost. Through simulations, authors demonstrated that the locally optimal scheduling scheme can achieve a performance close to the globally optimal scheduling scheme. Barco et al. [12], a methodology to plan the charging and the routes assignment for a BEVs fleet has been presented. This methodology considers the search of optimal routes and the minimization of operation costs. It has been found that the scheduling of charge and routes assignment have effects on the battery lifetime. A pattern for charging has been obtained, which allows increasing the battery lifetime. Floch et al. [13] developed and simulated a partial differential equation based model for grid-integrated plug-in electric vehicle (PEV) populations. This model can be utilized to optimally manage the PEV fleet to: (1) deliver contracted grid services, (2) supply drivers with sufficiently charged PEVs, and (3) minimize charging costs.

Acha et al. [14] presented a time coordinated optimal power flow (TCOPF) tool to illustrate the tradeoffs distribution network operators that might encounter when implementing various load control approaches of electric vehicles. The resolution framework is the stochastic dynamic programming, coupled with on-line minimization so as to avoid the curse of dimensionality[15]. The proposed resolution enables to compute optimal power flow for each vehicle, even among large fleets. Lan et al. [16] presented a mathematical formulation and a dynamic programming based algorithm for optimizing EV charging given electricity prices and driving pattern. With smart charging, EV is recharged during the lowest electricity price period, where is also the off peak hours which naturally drops the possibility of grid overload during the peak load hours. Iversen et al. [17] introduced an efficient stochastic dynamic programming model to optimally charge an electric vehicle while accounting for the uncertainty inherent to its use. With this aim in mind, driving patterns are described by an inhomogeneous Markov model that is fitted using data collected from the utilization of an electric vehicle. Foley et al. [18] introduced the impacts that EV charging can have in an actual working wholesale electricity market. EV charging profiles were produced for (I) off-peak and (II) peak EV charging scenarios. The results from these scenarios were analyzed to quantify the consequential effects of the additional EV load on the power system. Nguyen et al. [19] proposed charging strategies to reduce the charging cost of the charging station under time-varying electricity price signals. The random time arrival and random time departure of vehicle was taken into account. The performance of the proposed strategies was validated by simulations for a charging station of 50 electric vehicles in real time conditions. Vayá and Andersson [20] aimed to minimize charging costs while satisfying PEVs' flexible demand. To take driver end-use constraints into account the fleet is modeled as a virtual storage resource with power and energy characteristics that depend on vehicle behavior.

Wang et al. [21] considered a special EV network composed of fixed routes for an EV fleet, where each EV moves along its own cyclic tour of depots. By setting up a recharging station at a depot, an EV can recharge its battery for no longer than a pre-specified duration constraint. Authors presented an optimal deployment of recharging stations and an optimal recharging schedule for each EV such that all EVs can continue their tours in the planning horizon with minimum total costs. Lam et al. [22] studied an EV charging station placement problem for looking the best locations to construct charging stations in smart city planning. The authors gave a mathematical programming model with quadratic constraints and proposed four solution methods (iterative MILP, greedy approach, effective MILP and Chemical Reaction Optimization heuristics). Wang [23] proposed a set covering model with nonlinear constraints to address the electric scooter recharging facility location problem. It also considers the candidate sites, located on a given route or path of a single origin and destination (OD) and is validated on an island to recharge the recreation-oriented electric scooters for tourists. Wang [24] authors formulated a battery exchange station location model on a single path by a mixed integer program that can address multiple OD routing demands on that path which is based on [23]. Wang and Lin in [25] further extended research to calculate a minimum cost recharging facility location problem that considers intercity OD round-trips. A hybrid model with dual objectives of minimum cost and maximum coverage was formulated by Wang and Wang in [26]. The presented hybrid model could account for the travel of both inter-city and intra-city distances. MirHassani 
and Ebrazi [27] formulated a flexible MIP model based on the assumptions presented by Kuby and Lim [28]. The presented model was more efficient than previous set covering models. Hosseini and MirHassani in [29] considered the traffic flow uncertainty in determining the locations for both permanent and portable refueling stations. Hosseini and MirHassani in [30] considered the waiting time to build recharging stations at capacitated recharging stations for given locations. If the number of EVs exceeded the station capacity, a queue is formed.

In summary, our paper differs from previous works in the aspect of calculating the detailed optimal recharging plans in addition to the optimal location decisions. We have integrated the activity based modelling to calculate the proportion of population which can be served by the electric vehicle recharging model. Using the operational activity based model for Flanders region in Belgium, we used realistic trip data to find the time based usage of quick charging stations.

\section{Simulation model for electric vehicle charging}

A simulation tool is described in this paper which calculates charging requirements of BEVs depending upon their travel demand then plans a charging strategy to fulfil the travel requirements. The simulation model inputs the daily travel schedules planned by an activity-based model and evaluates the feasibility of schedules which can be executed using the electric vehicles. Each schedule has different characteristics in terms of number of home-tohome tours, trips in each tour and charging time when vehicle is parked at home or at work. If a vehicle is sufficiently charged while parking at home/work, this is the most efficient solution which does not lose any time. If home and work charging is insufficient, then a charging station is selected during a trip in a tour to charge the battery using fast chargers. This results in time loss that is compensated in the last home activity in the schedule.

A conceptual framework is presented in the next section which describes the working of the model; it describes different modules, their functions and the interaction among these modules. The next subsection provides a more technical solution that is used to implement the model. It presents the architecture of the model along with technical details.

\subsection{Conceptual framework}

A conceptual overview of the framework is expressed in the Figure 1, which shows the principle of operation at an abstract level. There are four main parts in the system. 1) EV Agent 2) Quick charging station (QCS) Selector that is part of EV Agent but has its unique role 3) QCS use administrator and 4) power consumption tracker. Each electric vehicle in the system is represented by a unique EV Agent which uses the planning algorithm to devise the charging strategy. EV agent fetches its daily travel schedule and determines whether all of the travels can be completed if the vehicle is charged only at home and if possible work locations. It is assumed that each electric vehicle owner has a charging facility at the home location. In addition to this, a predefined fraction of the EV users having a work activity can charge at their work premises. A detailed statistic on modelling the charging facilities and infrastructure for experiment is described in section 4.1. If charging at home and at work is insufficient, then EV agent uses the QCS selection module which interacts with QCS use administrator module to get information of location, occupancy rate and availability of quick chargers at all quick charging stations. Depending upon the travel time and distance required to travel to quick charging station, waiting and charging time, the QCS selection module selects the optimal station with minimum time loss. Using the selected QCS the EV agent updates the schedule to compensate the lost time due to charging activity. Power consumption tracker keeps track of total energy consumed by the electric vehicles for charging either at home, work or quick charging stations. 


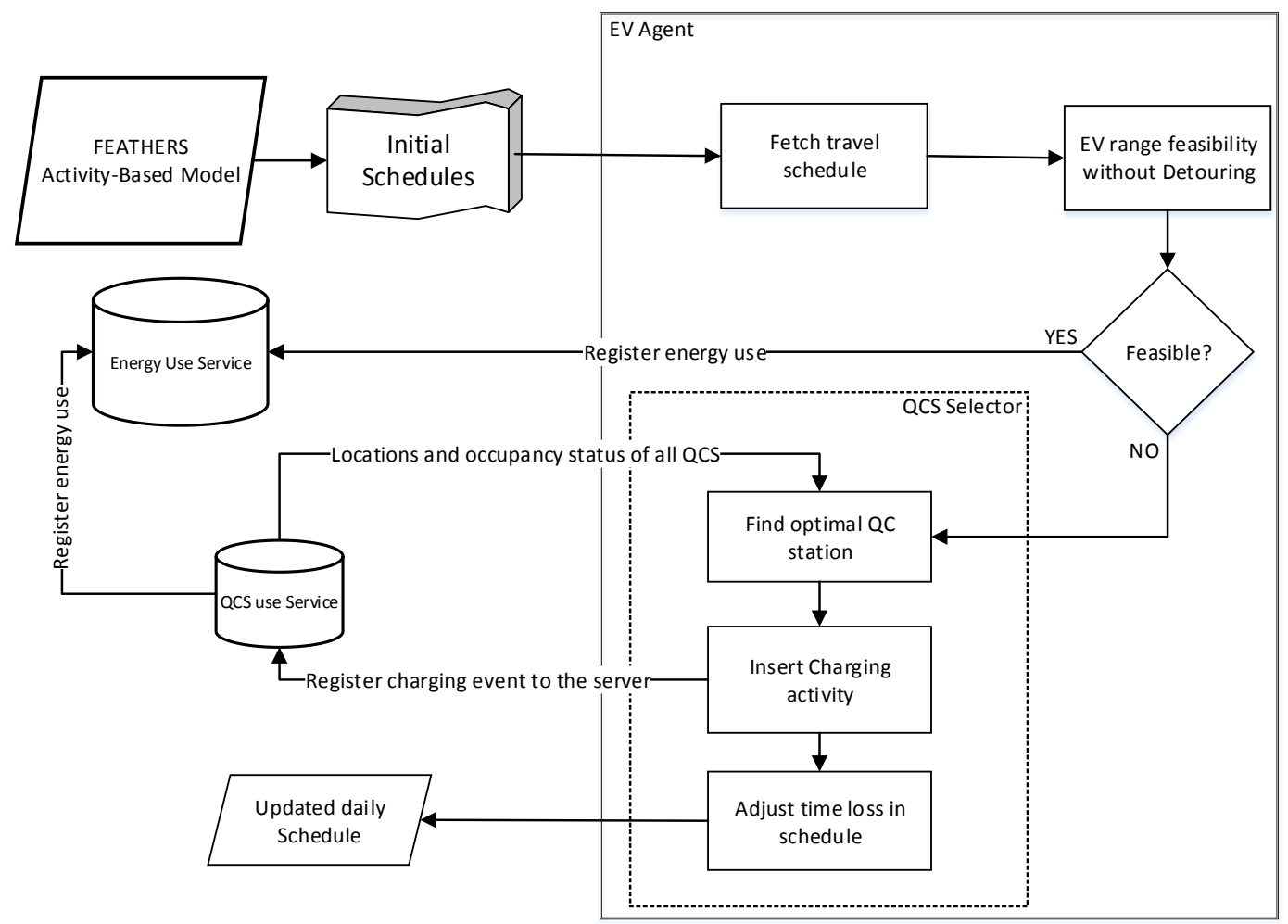

Figure 1 conceptual framework for recharging of electric vehicle

\subsubsection{Daily travel schedules}

Daily travel schedules planned by an activity-based model are used as input to this model. The initial schedule for every inhabitant of Flanders (Belgium) is generated by the FEATHERS; an activity-based model described in [31]. In the FEATHERS context a schedule is a sequence of episodes for a period of $24 \mathrm{~h}$. Each episode consists of a journey (trip) and an activity.

$$
\begin{aligned}
& \langle\text { Schedule }\rangle:=\langle\text { Episode }\rangle * \\
& \langle\text { Episode }\rangle:=\langle\text { Trip }\rangle\langle\text { Activity }\rangle
\end{aligned}
$$

The trip is characterized by a tuple (origin, destination, start time, duration, mode). The activity is characterized by a tuple (activity type, location, duration). All trip attributes except for the mode can be derived from the consecutive activities in between which it is enclosed. For each member of the synthetic population (also called a user), a schedule is predicted.

The first and last activities in the schedule are always home activities. There can be one or more intermediate home activities other than the first and last home activities in a schedule. A home-to-home tour is a sequence of episodes where, except for the first and last ones, no intermediate home activity occurs. Hence, a schedule consists of one or more home-to-home tours. Note that the first home activity of the day has a zero trip.

\subsubsection{EV Agent}

Each electric vehicle contains an independent virtual object called EV agent. It plays central role in planning charging strategy for electric vehicle. When a user registers its day-ahead travel schedule, it optimizes the charging strategy and updates the schedule to adjust it to any time loss occurring due to stopover at a quick charging station. 
Stopover at quick charging station is only considered if charging at home and or work is not sufficient to complete the all schedules trip in a day. Hence, EV agent completes the charging planning in two steps.

I. Charging at home and if possible at work

II. If charging at home and work is insufficient then charging at one of the quick charging stations.

\subsubsection{Charging planning process without detour}

Upon receiving the schedule for the next day (24 hours), EV agent starts planning the charging process. The first step of the process is to evaluate if charging only at home or work location is feasible to use EV for a tour. Suppose that a car $C$ receives its traveling schedule $S$ of a day $D$. A day $D$ consists of 96 periods $p$ where each period is 15 [min] long. It is assumed that Car has its battery SOC at some INITSOC level at start of the day. For all trips $T$ in each tour of schedule $S$ where car $C$ is used as transport mode, the planner ensures that the car battery is charged enough so that it does not go below to the minimum level during the trip. $D C D$ (deepest charge depletion) level of the battery is used as the minimum threshold for battery SOC for all trips. In case of battery SOC goes below to the minimum level after a particular trip, a time slot is selected between two time periods $t_{0}$ and $t_{1}$, where $t_{1}$ is the last period before the trip and $t_{0}$ is the last period when the battery was full. Similar to period $p$, a 15 [min] time window is used to for the terms of time slot in this optimization process. In case of no battery full event found, starting period of the tour is used as $t_{0}$. The first slot from the list that fulfills the following conditions is selected to charge the car:

1. The vehicle is not traveling during the complete period associated with the time slot. It should have some parking moments during the period at a location where a charging is possible.

2. The time slot is not already booked for this vehicle.

If any slot is found, the planner first determines the energy that can charged during the slot. It depends upon the power of the charger that will be used for charging at the found time slot. Therefore, the planner first determines the location where car will be parked at the found time slot (i.e. either at home or at work). Then, it determines the vehicle presence time at this location overlapped with period of found slot. Using the presence time and charger power it calculates the maximum energy $E_{\max }$ that can be charged at this particular slot. Then it calculates the effective energy that is planned to charge at this slot by taking the minimum of required energy to meet minimum energy level constraint, the amount of energy that can be charged during this slot and the amount of the energy that can be charged before the battery gets full during already planned charging in successive slots. This effective energy is added to the battery SOC. If this optimization process successfully iterates over all trips to keep the battery SOC above minimum level at each point in time, this schedule is marked as "feasible". In case charging at all slots where a charging facility is available is insufficient to travel all the trips, the schedule is marked as "infeasible". In case a feasible charging pattern is found, information about charging events is sent to register at power consumption tracker. In case of infeasible situation, EV agent uses its QCS selector module to make a stopover at any QCS station to charge during the tour. Section 3.1.4 provides complete description on design and working of QCS selector module.

Algorithm 1 shows the main components of the charging planning process without a detour used by the EV agent. Lines 1-5 perform general initialization by receiving the inputs. Lines 6-31 determine the optimal set of charging slots. Lines 34-41 specify the required minimum SOC at the end of trip $T$ before the next trip is processed.

\subsubsection{QCS Selector}

Quick charging station (QCS) is a fast charging facility installed on a particular location; it contains a depot of fast DC chargers. A QC station has a fixed number of chargers of identical power [kW]. Hence, each QC station is characterized by its location, the number of chargers installed on the premises and the charging power.

The quick charger selection module selects the QCS out of all reachable QC stations to be used for charging during a trip by the EV which yields minimum time loss. Reachable QCS stations are those which can be reached by the EV during a trip with given the SOC at the start of the tour. The QCS selections module interacts with the $Q C S$ use administrator to get information about available QCS, their location, already booked charging events and 
waiting requests for each minute of the day. A charging event contains information about charging starting time, charging duration and charged energy during the event.

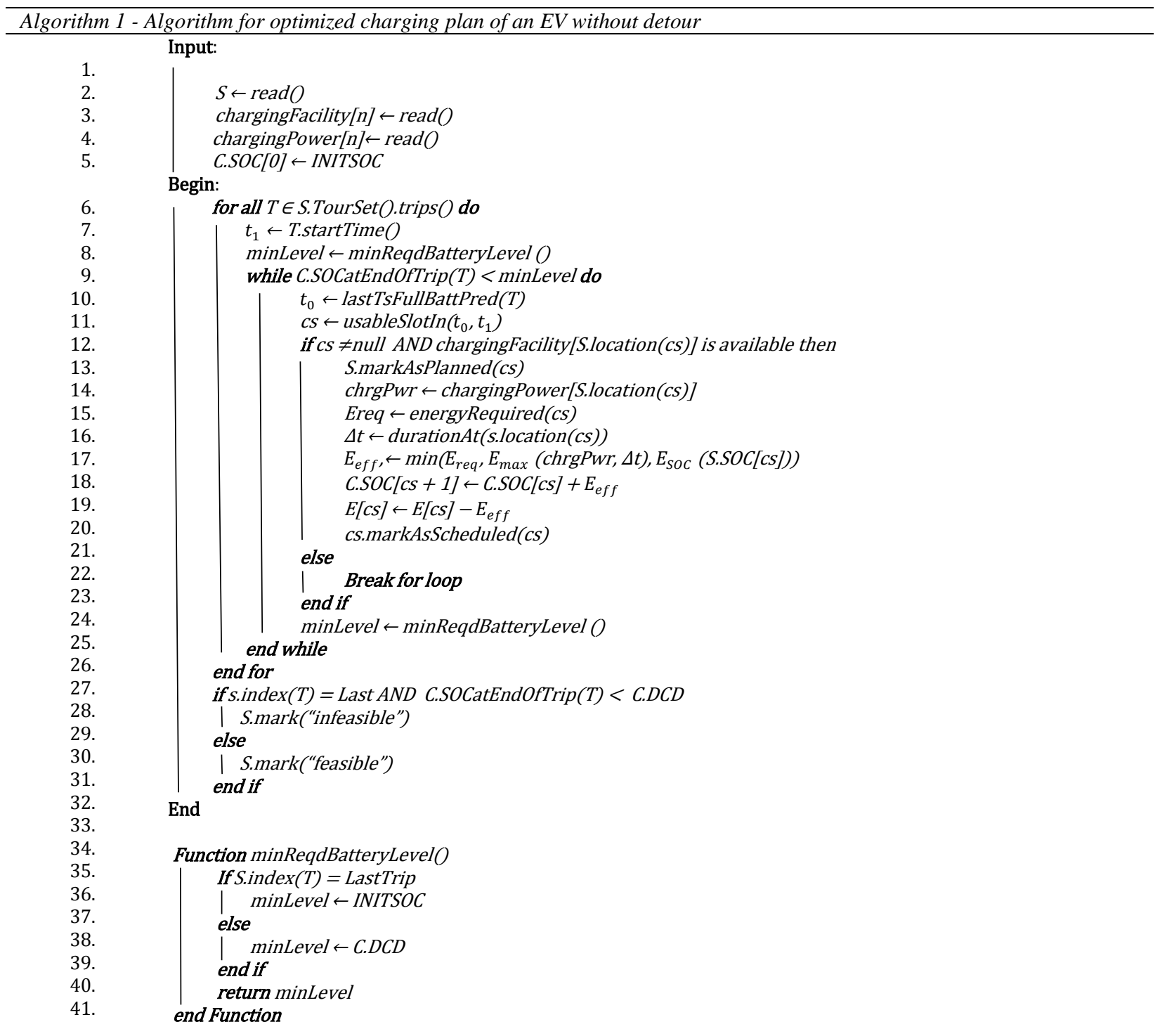

An example of a schedule where a charging activity has to insert is shown in the Figure 2. A schedule consists of 8 activities and 7 trips in between them. There are two home-based tours. The first tour consists of 5 activities (Home-Pick/Drop-Work-Shopping) and 4 trips with numbering from 1 to 4 . There are two reachable QCS stations where QCS1 can be used during trip 2 and QCS2 can be used during trip 3. Out of 2 QC stations, $2^{\text {nd }}$ QCS is selected because total time required to use QCS2 (15 $\mathrm{min})$ is less than that of QCS1 (18 $\mathrm{min})$. 


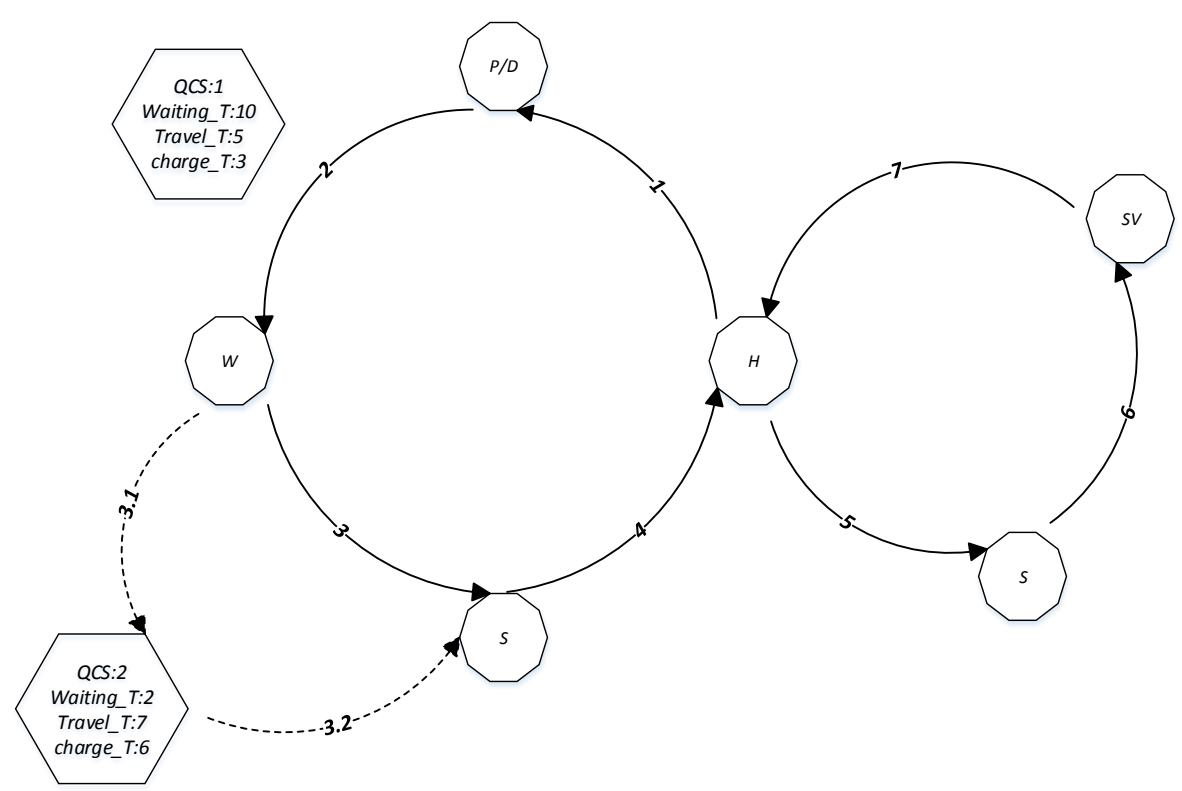

Figure 2 - An example to select a QCS with minimum time loss

QCS selector being part of the EV agent already contains the daily schedule $S$ of the user. It also contains the information of the SOC level at the start of each home-based tour (HH tour). Assume that schedule $S$ contains $h$ number of $\mathrm{HH}$ tours, where each tour $H$ starts with a unique INITSOC[H]. Each tour $H$ consists of $t$ trips where departure location of first trip is always same as arrival location of last trip which is home. Each trip $T$ in tour $H$ has its departure location, arrival location, distance, starting time of the trip and its travel duration. If QCSList contains all $Q C S$ in study area, then $Q C S$ selector module selects one qcs station using following steps:

Starting the tour with initial SOC, it lists determines all reachable (candidate) QCS stations during each trip. Assume that candidateList $[T]$ contains the reachable QCS during trip $T$ in tour $H$. Each car $C$ is modelled so that it has its unique energy consumption rate $[\mathrm{kWh} / \mathrm{km}]$; further details about electric vehicle technical characteristics are described in section 4.1.2. A qcs from QCSList is added to candidateList for Trip T if condition (3.4) holds.

$$
\begin{aligned}
& \text { range }=(\operatorname{INITSOC}[H]-D C D) / \text { EnergyCons }(C) \\
& \sum_{i=0}^{\text {index }(T)-1} \operatorname{distance}\left(\operatorname{Trip}_{i}\right)+\operatorname{distanceMatrix}[\operatorname{departLoc}(T)][q c s] \leq \operatorname{range}
\end{aligned}
$$

Then it prunes the candidate list by discarding the QCS from the list of each trip which are infeasible to complete the tour if user departs from that particular QCS with full battery. If car $C$ has battery capacity batCap, range of the vehicle is calculated as shown in equation(3.5). Starting with full range of the vehicle, any qcs in candidateList of QCS for a trip $T$ is thrown away if condition (3.6) does not hold.

$$
\begin{aligned}
& \text { range }=\operatorname{batCap}(C) / \text { EnergyCons }(C) \\
& \text { range } \geq \operatorname{distanceMatrix}[q c s][\operatorname{arrivalLoc}(T)]+\sum_{i=i \operatorname{ind}(T)+1}^{t} \operatorname{distance}\left(\operatorname{Trip}_{i}\right)
\end{aligned}
$$


QcsSelector enumerates over the feasible list of QCS to calculate the travel time, waiting time and charging time. Hence, the excess trip duration caused by the requirement to charge at each qcs in candidateList for each Trip $T$ is shown in equation(3.10). Waiting time at the qcs station is calculated as difference in arrival time at $q c s$ and first consecutive availability of any charger at the qcs for chargingTime [min] duration.

$$
\begin{aligned}
& \operatorname{disTravelled}(T, q c s)=\sum_{i=0}^{\operatorname{index}(T)-1} \operatorname{distance}\left(\operatorname{Trip}_{i}\right)+\operatorname{distanceMatrix}[\operatorname{departLoc}(T)][q c s] \\
& \operatorname{reqCharging}(T, q c s)=\operatorname{batCapacity}(C)-\operatorname{disTravelled}(T, q c s) * \operatorname{energyCons}(C) \\
& \text { chargingTime }(\text { power, reqCharging })=\text { power } / \text { reqCharging } \\
& \text { time }[T][q c s]=\text { TravelTime }[\operatorname{departLoc}(T)][q c s] \\
& \text { +TravelTime[qcs][arrivalLoc }(T)] \\
& \text { +waitingTime (qcs, reqCharging (T, qcs), arrivalTimeAtQCS) } \\
& + \text { chargingTime ( power }(q c s) \text {, reqCharging }(T, q c s) \text { ) } \\
& - \text { duration }(T)
\end{aligned}
$$

The QCS that has the minimum time required is considered optimal qcs to be used for charging during the tour.

$$
\operatorname{optimal}(q c s) \leftarrow \operatorname{Min}_{T \in \operatorname{Tr} p(H)}^{q c s \in \text { candidateList }(T)} \operatorname{time}(q c s)
$$

After a selection of QCS, the initial schedule is updated and lost time is accounted. The trip $T$ during which an optimal qcs is found, is decomposed into two trips $T_{1}$ and $T_{2}$ and a charging activity $A_{c}$ is inserted in between the trips. The replacement of a trip with two trips and a charging activity increases the total schedule duration that is adjusted by decreasing the duration of last home activity. Assume that $A_{h}^{d u r}$ is the last home activity duration in the schedule, then new duration is calculated using following equation.

$$
A_{h}^{d u r}=A_{h}^{d u r}-\left(T_{1}+A_{c}^{d u r}+T_{2}-T\right)
$$

An example is shown in the Figure 3 to illustrate the insertion of a charging activity in the schedule. Consider a charging activity that was required to complete the tour from home activity $A_{0}$ to the home activity $A_{3}$ which requires to travel the trips $t_{1}, t_{2}$ and $t_{3}$. Assume that the optimal location for charging is found during the trip $t_{2}$. Hence, a charging activity $A_{c}$ has to be inserted during the trip $t_{2}$ that will be replaced by 2 trips $t_{21}$ and $t_{22}$; first from previous location to the charging station and second from charging station to the next activity. When trip $t_{2}$ is replaced by two trip and charging activity, it will shift the succeeding activities to the future that eventually increases the day length. Hence, increased total schedule duration is adjusted into last home activity. 
Algorithm 2 shows the steps used for charging station selection and updated the schedule after charging activity insertion. Line 4-24 show the method of filtering charging station, which can be used for quick charging during the travel. Line 26 shows how to find the optimal QCS out of all candidates in the list. Lines 26-30 describe the steps for inserting the charging activity and schedule update.

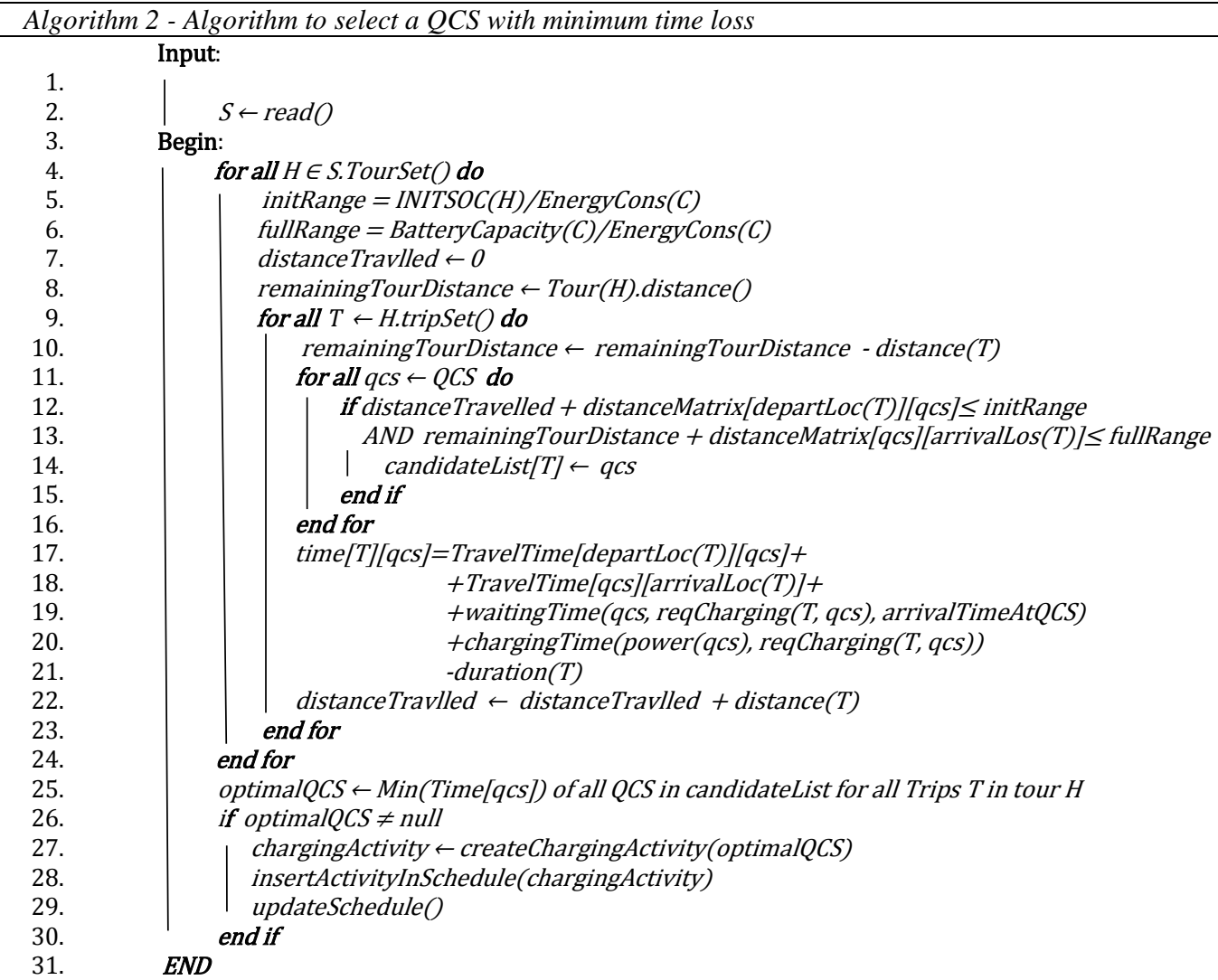

\subsubsection{QCS use administration service}

This service manages the use of charging stations to ensure that none of the station is overbooked. It keeps track of bookings made for each station. Depending upon the current booked status, this service also provides the waiting time for any new charging request at given station for given duration at any time of the day. In this study, we assume that qcs administration service manages all qcs stations in the study area and provides communication channel to receive charging requests from $E V$ agents, provides the response back to them, and to communicate

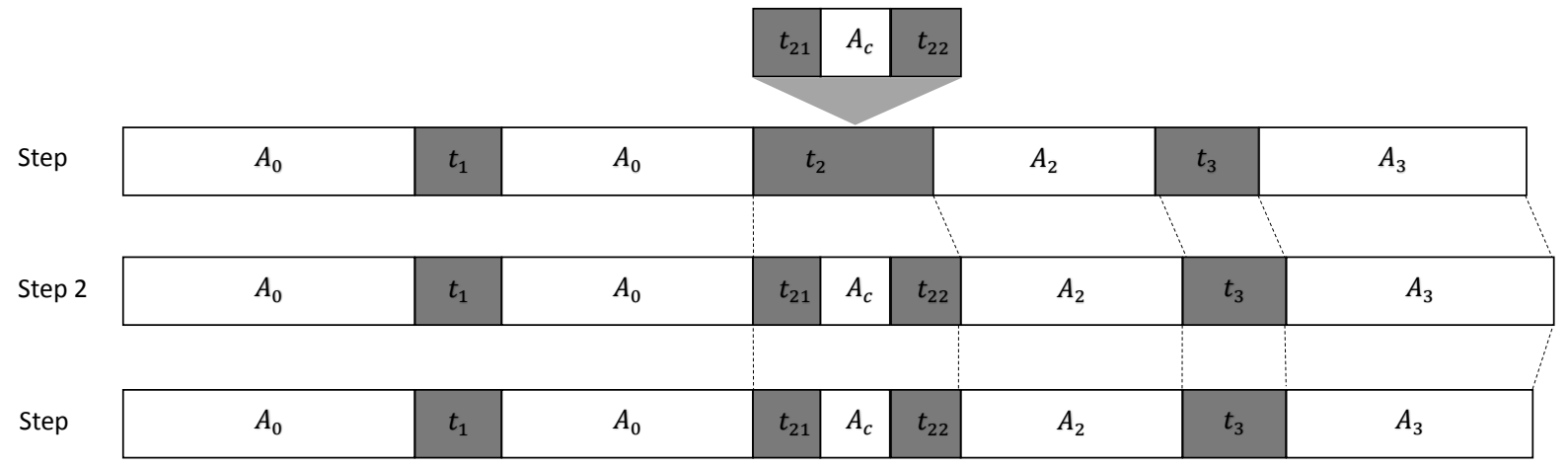

Figure 3 - An example to insert a charging activity in a schedule and update the lost time 
with all charging stations as well. This administration service keeps track of all completed and active occupation and registered request for the complete day at all stations.

During the process of finding optimal charging station, QCS selector sends requests to administration service for each qcs in candidateList with the amount of energy it wants to charge and the arrival time at qcs. The administration service calculates and replies with waiting time at each qcs to charge the required energy. QCS selector finds the optimal charging station having minimum total activity time which includes travelling, waiting and charging time, and sends another request to administration service to register the charging request with given optimal charging station. The service registers the requesting vehicle at given charging station by registering the assignment of charging place at given QCS to the vehicle.

Assume that there are $n$ charging stations where each charging station has $m$ charging points. Each request to get waiting time contains $q c s$, energy (eng) to be charged and time of arrival $\left(t_{\text {arr }}\right)$ at the station. Since each QCS station contains $m$ charging points, it keeps record of occupation of each charging point at the station for the complete day. Charging point can have either available or occupied status for each minute of the day. At the start of the day, each charging point at all QC stations is set as available. When a new charging request is assigned to a charging point at a given QCS, it is set as occupied from charging start time to finish time. To evaluate the waiting time to get an available charging point to start delivering energy, the administration service first calculates the time a charging point $i$ will take to deliver the required energy, then it computes its earliest consecutive availability equal to charging duration. This difference in earliest availability and arrival time at qcs is the waiting time when charging point $i$ is considered. Then for each QCS stations, it takes the minimum waiting time of each charging point $i$, and sends this back to requester. Algorithm 3 shows the functions to calculate the waiting time and assigning a charging request to a charging point.

\section{Algorithm 3 - Algorithm to calculate waiting time and assigning a request to charging point}

1. $\quad$ Function getWaitingTime(qcs, eng, arrivalTime)

2. $\quad$ for all $i \in$ qcs.chargingPointsSet do

3. $\quad$ chargingDuration $\leftarrow$ eng/i.power()

4. $\quad$ for all t in [arrivalTime, minutesInDay - chargingDuration]

5. $\quad$ if i.isAvailable(t, chargingDuration)

6.

7.

8.

9.

10.

11.

12.

13.

14.

15.

16.

17.

18.

19.

20.

$$
\text { | Break for loop }
$$

\subsubsection{Power consumption tracker}

Power consumption tracker keeps record of used energy for charging electric vehicles at any location; at home, office or charging station. When a feasible charging plan is found, the power consumed is registered for statistics purpose. If a charging plan is found without visiting a charging station, EV agent send the consumption to energy user service. Each consumption record contains the location, consumed power with starting and finish time stamps. Similarly, when a charging station serves any vehicle, it also sends the consumption data to the energy use service.

\subsection{Simulation Implementation and technical details}

A software simulation is implemented using Java to experiment the theoretical framework described above. Daily activity schedules of individuals, which are used as source input to feed the model are created by the FEATHERS 
activity-based model. An individual may have a conventional combustion engine vehicle or a battery only vehicle. All those individuals who have BEV are used as input to the model.

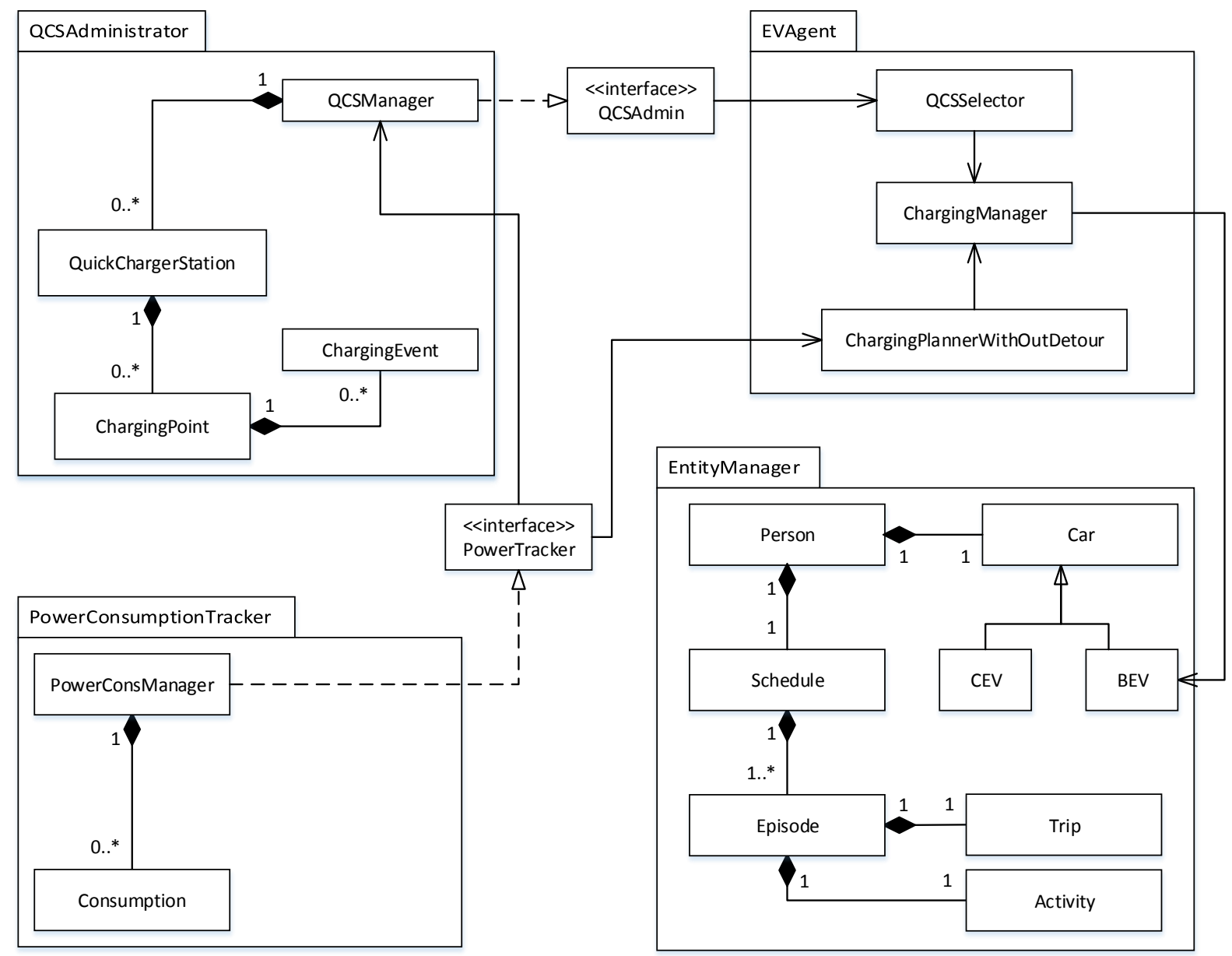

Figure 4 - UML class diagram of the proposed simulation model

Software is implemented using four modules that are bridged using interfaces. The entityManager module provides the model of input to the software. It mainly contains the list of Person objects. Each Person object has information about the household attributes (i.e. location, household composition), one Schedule object and one car object. The car can be either a combustion engine vehicle $(C E V)$ or a battery only vehicle (BEV). Each $B E V$ vehicle has attributes of current state of charge $(S O C)$, its energy consumption rate per unit distance, battery capacity, and its deepest charging depletion level. Each Schedule has many Episodes, where each Episode object has one Trip and one Activity. Each Trip has its departure time, duration, departure location, arrival location, and transport mode. Each Activity object has its starting time, duration, location, and type of the activity. The next important bundle is QCSAdministrator, which has a class QCSManager to manage the use of all charging stations. QCSManager has many QuickChargingStation objects. Each QuickChargingStation object has a location and list of ChargingPoint objects. Each ChargingPoint object has its charging power [kW] at which it can deliver energy and a list of ChargingEvent objects. Each ChargingEvent has information about charging start time, duration, delivered energy and a reference to vehicle that uses the event. Another module is PowerConsumptionTracker 


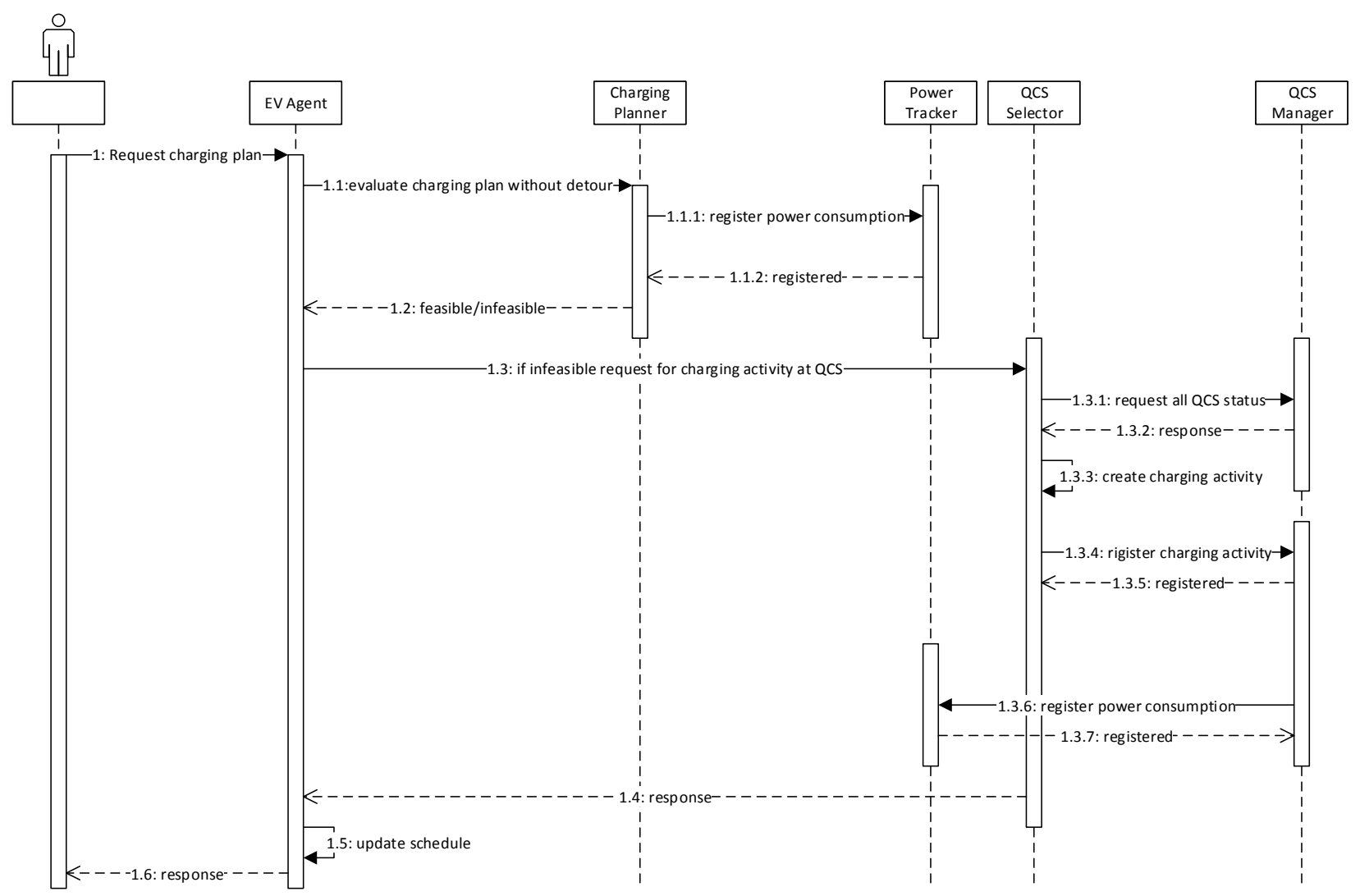

Figure 5 - UML sequence diagram of the proposed simulation model

that has PowerConsManager to keep track of used electricity for BEVs at any location (i.e. at home, work, or charging station). Each PowerConsManager object contains a list of Consumption object where each object of Consumption contains information of one charging activity with details of charging start time, duration and location. The most important bundle EVAgent contains a ChargingManager and provides two classes for the charging planning process. ChargingPlannerWithoutDetour provides the implementation of finding suitable charging strategy if vehicle can execute all of its trips with charging only either at home or at work places. On the other hand, the QCSSelector class provides the implementation to find the optimal location of quick charging station if vehicle have to make a stop during a trip in the tour to recharge battery. Figure 4 shows the UML class diagram of all modules in the software.

Figure 5 shows the UML sequence diagram of interactions that occur between different entities in the application. When an individual has his schedule for next day and it has at least one tour using $B E V$ transport mode, $B E V$ starts the charging planning process. $B E V$ sends a request to ChargingManager to find a charging plan for given schedule. ChargingManager first sends a request to ChargingPlannerWithoutDetour to find a charging plan by charging only at home and work locations. If this module finds a feasible plan, it requests to PowerConsumptionTracker to register the consumptions. Then it sends a response back to ChargingManager. If the response was infeasible, ChargingManager sends a request to QCSSelector to find an optimal QCS for charging. QCSSelector sends a request to QCSManager to get waiting time to get charging point at given QCS. After the response is received, QCSSelector finds the optimal QCS and request to QCSManager to register the charging activity at given QCS for given time and duration. QCSManager requests to PowerConsumptionTracker to register the consumption for newly registered charging activity. QCSSelector sends the response back to ChargingManager with found charging activity. ChargingManager inserts the charging activity into the schedule and update the starting time of all succeeding activities. At the last step, ChargingManager sends an updated schedule back to $B E V$. 


\section{Simulation experiment and results}

\subsection{Input data}

\subsubsection{Tours characteristics}

As described earlier, daily travel schedules used to calculate travel demand are created by FEATHERS an activitybased model. FEAHERS create daily schedules of 2395514 schedules, out of which 1942497 schedules contain at least one car tour. Figure 6 shows the frequency of tours for different ranges of distance. On the x-axis, it shows the distance range of different bins and on y-axis it shows the frequency of the tours which belong to the particular distance range. From the figure, it is clear that distances of more than $50 \%$ of tours are less than $30 \mathrm{~km}$. On the other hand, almost $3 \%$ of trips are more $200 \mathrm{~km}$ long.

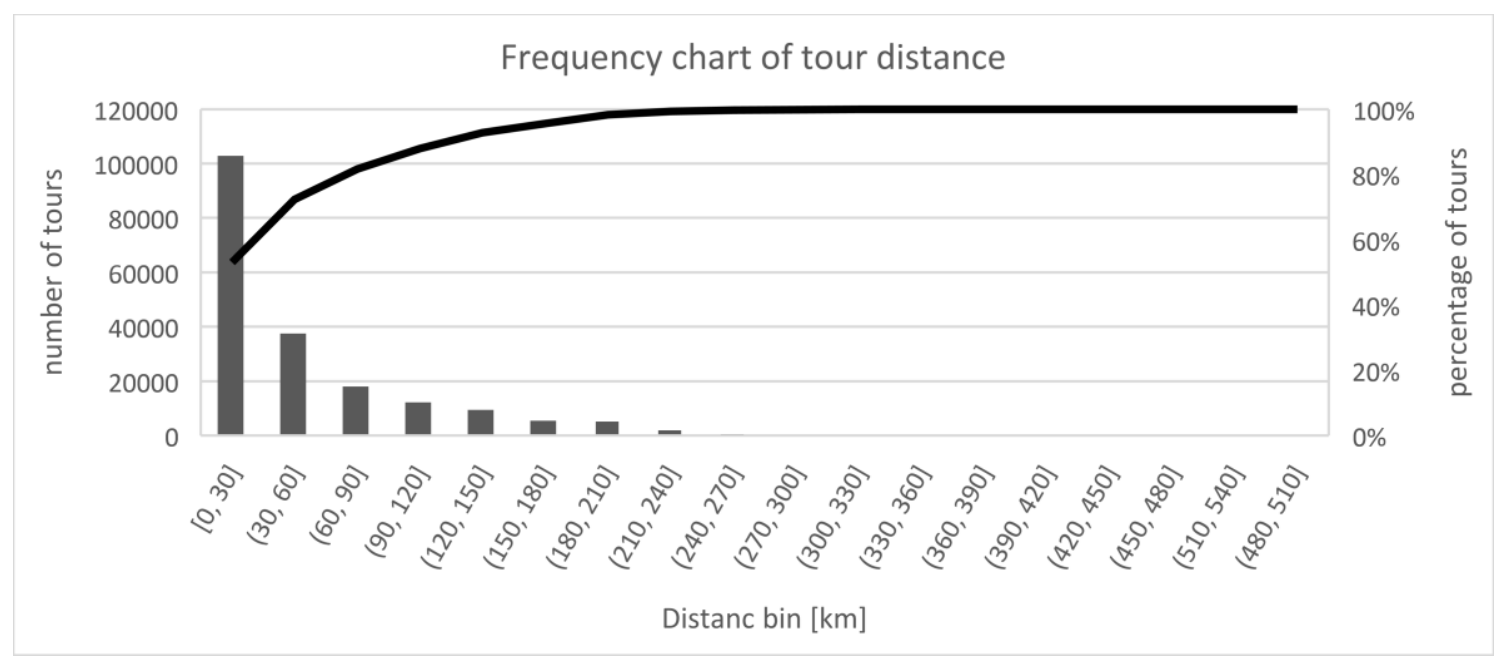

Figure 6 - frequency distribution chart of tour distance

\subsubsection{Electric vehicle categories}

Electric cars are subdivided into the categories small, medium, large similar to what is done in [32]. In order to estimate the energy requirement, one needs to know the contribution of each one of those categories to the complete vehicle set similar to what is done in [33] [34] [35]. Belgian government statistics provide a classification of internal combustion engine (ICE) vehicles based on the motor cylinder volume: they provide a distribution of the registered cars using that classification. We state the one-to-one mapping of categories given in Table 1 that shows market share and technical characteristics for each category. Vehicle characteristics in the table have been derived from data in [32] and [36], the market share figures have been taken from the Belgian federal government 2009 statistics (PARC010 Transport Indicator) [37] Taking market share for BEV of 10\% of the total vehicle fleet, we sample 194249 schedules from the complete set using a uniform distribution.

To charge EVs at home or work places two types of chargers are considered: 3.3 [kVA] and 7.2 [kVA] chargers compatible with the Flemish grid. Our model distinguishes between home and work location chargers. The power value for home chargers is assumed to depend on the car category: smaller cars are equipped with a less powerful charger. On the other hand, companies offering car charging facilities provide powerful chargers in order to save time and extend the distance that can be bridged during one day. In Belgium, employers are believed to allow company car (CC) drivers to charge at the work location because that is less expensive than providing fuel cards to employees. However, for technical reasons, some companies cannot provide the required infrastructure. The 
fraction of actors who can charge batteries at the work location has been determined as a fraction of company car drivers. It has been assumed that $50 \%$ of the work locations provides suitable infrastructure for battery charging.

Table 1 - Technical characteristics of electric vehicle categories and charging equipment at home and work

\begin{tabular}{|l|c|c|c|}
\hline & \multicolumn{3}{|c|}{ Vehicle categories } \\
\hline Equivalent engine cylinder volume [cc] (ICEV category) & V $<1400$ & $1400 \leq \mathrm{V} \leq 2000$ & $2000>\mathrm{V}$ \\
\hline Market share (from Belgian government statistics) & 0.496 & 0.364 & 0.140 \\
\hline EV category & small & Medium & Large \\
\hline Battery capacity $(\mathrm{kWh})$ & 10 & 20 & 35 \\
\hline Range $(\mathrm{km})$ & 100 & 130 & 180 \\
\hline Energy consumption $(\mathrm{kWh} / \mathrm{km}):$ lower limit & 0.090 & 0.138 & 0.175 \\
\hline Energy consumption $(\mathrm{kWh} / \mathrm{km}):$ upper limit & 0.110 & 0.169 & 0.214 \\
\hline Charger type at home : Prob $(3.3[\mathrm{~kW}])$ & 0.8 & 0.4 & 0.1 \\
\hline Charger type at home : Prob $(7.2[\mathrm{~kW}])$ & 0.2 & 0.6 & 0.9 \\
\hline Charger type at work : Prob $(3.3[\mathrm{~kW}])$ & 0.1 & 0.1 & 0.1 \\
\hline Charger type at work : Prob $(7.2[\mathrm{~kW}])$ & 0.9 & 0.9 & 0.9 \\
\hline
\end{tabular}

\subsubsection{Quick Charging stations}

Each quick charging station has a pool of quick chargers where charging power of each charger may vary. Quick chargers are rated as level III chargers in technical categories. Level III or quick chargers have charging power from $43 \mathrm{~kW}$ to $50 \mathrm{~kW}$. Using $50 \mathrm{~kW}$ charger, an electric vehicle having large battery of $35 \mathrm{kWh}$ can be recharged completely in 42 minutes only. 11 quick chargers which are installed in Belgium are used to test the described simulation tool. Information about location of quick charging stations is taken from [37] which provides independent and real-time view of all charging stations in specified region [38]. Figure 7 shows the location of all 11 quick chargers used in this study. It is worth to note that all chargers are installed in vicinity of Brussels region while travel schedules cover the complete Flanders region. Hence, it may become infeasible to use these chargers for those EV drivers who only travel in far east and west areas from Brussels.

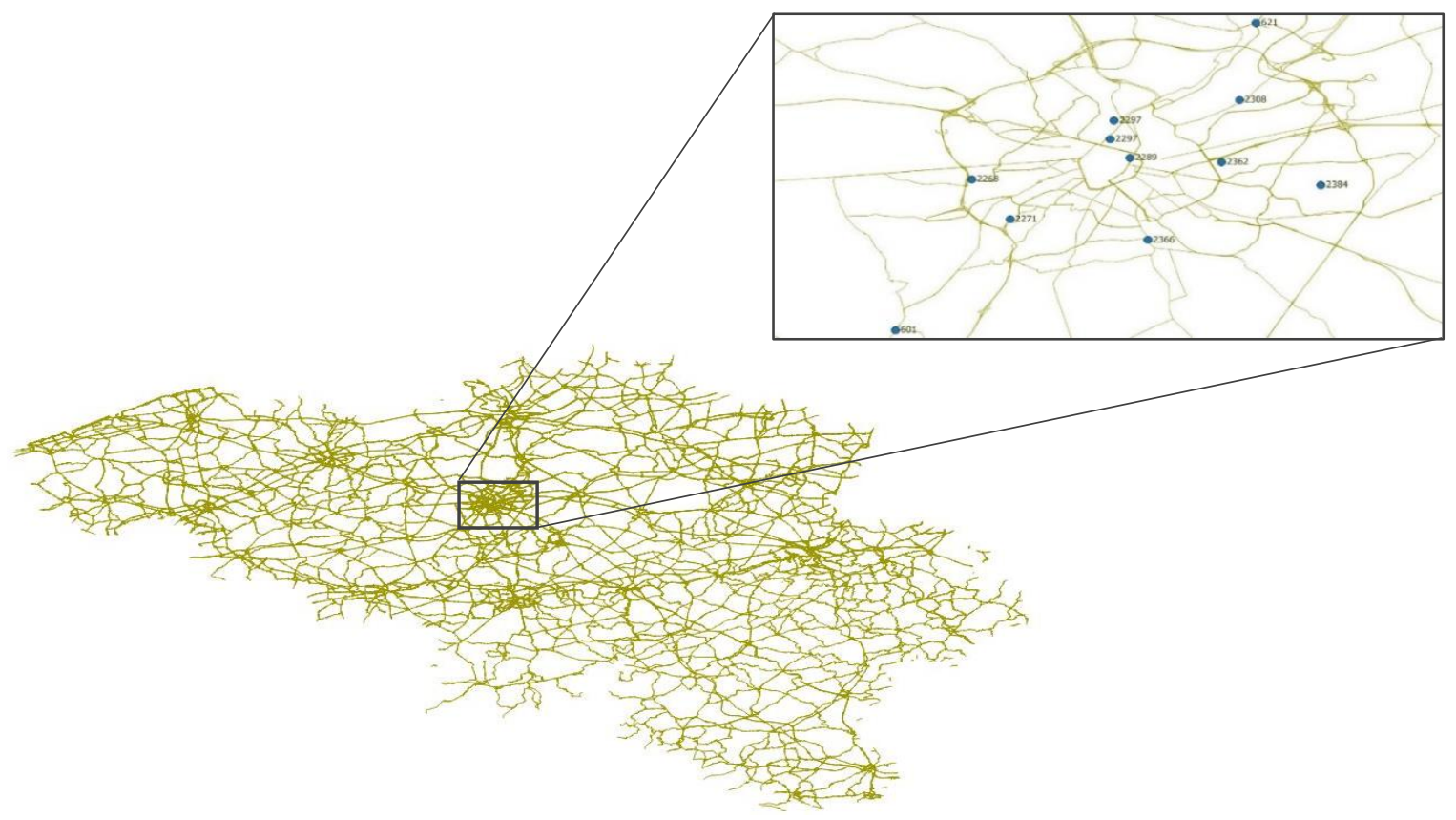

Figure 7 - Geographic locations of quick charging stations in Flanders 


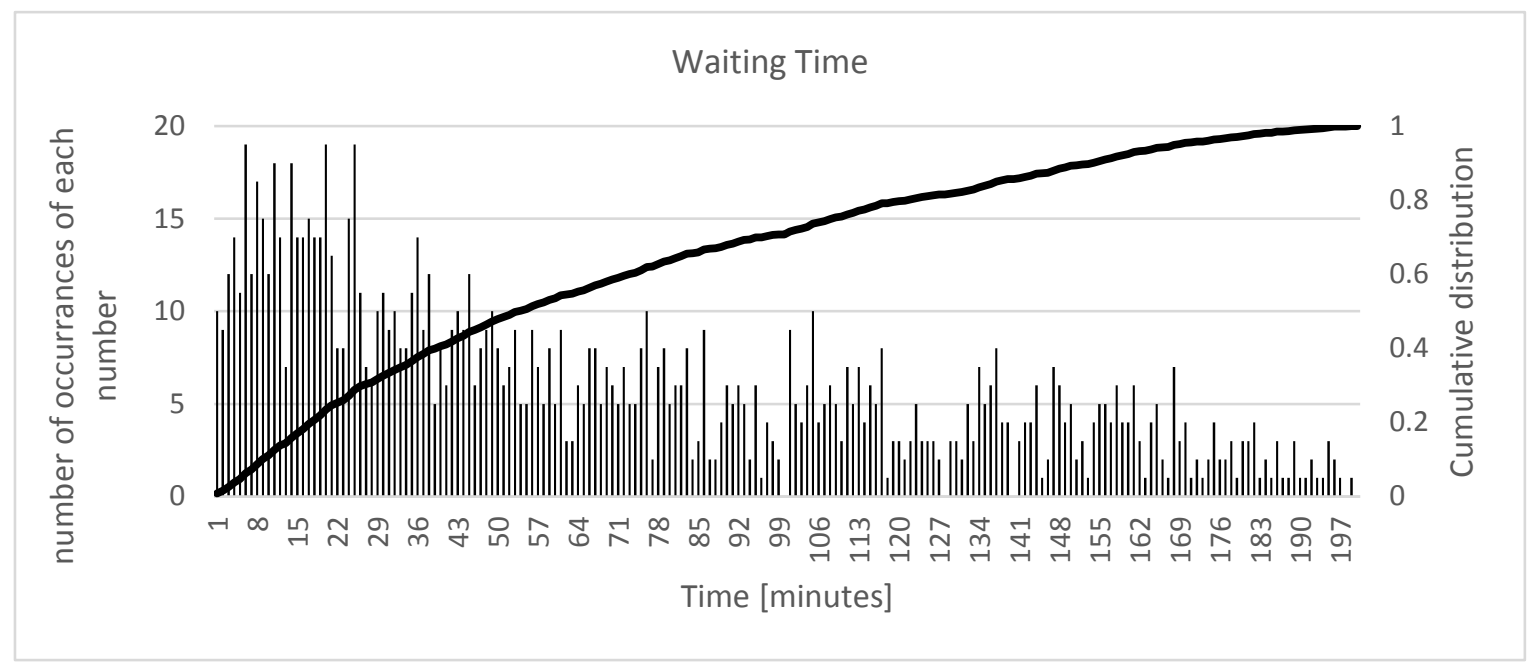

Figure 8- Frequency distribution of waiting time for electric vehicles at all charging stations

\subsection{Experimental results}

According to the scenario used to test the simulation, all 11 charging stations are assumed to have 25 charging points where each charging point can deliver energy with $40 \mathrm{~kW}$ power. Waiting time at QC station depends upon the number of vehicles that have already booked a charging slot at give charging station. Figure 8 shows the frequency of waiting time on all charging stations. An upper bound of the waiting is used as 200 minutes. Although waiting for a long duration is not realistically compatible, for simulation we used this number just to add an upper bound constraint. In figure is apparently visible that waiting time of around $50 \%$ requests is up to 50 minutes. Waiting time is a function of installed charging points on each charging station. Changing the number of charging points on each $Q C S$ will generate different waiting time distribution.

Figure 9 shows the distribution of total time lost after inserting a charging activity into the schedule. Time lost contains the waiting time, charging time and difference in travel time of replaced original trip with two new trips bridging the original departure and arrival location with charging station. Looking at the figure, it is apparently visible that around $50 \%$ of the charging request lose up to 60 minutes of time from the schedule.

Figure 10 shows the occupancy of all 25 charging point on 8 charging stations. All parts of the figure show the time of the day [minute] on $\mathrm{x}$-axis while $\mathrm{y}$-axis represents the number of occupied charger at each minute during the day. The day starts from 03:00 am (180 [minutes]) in the morning and ends at the same time (1620 [minute])

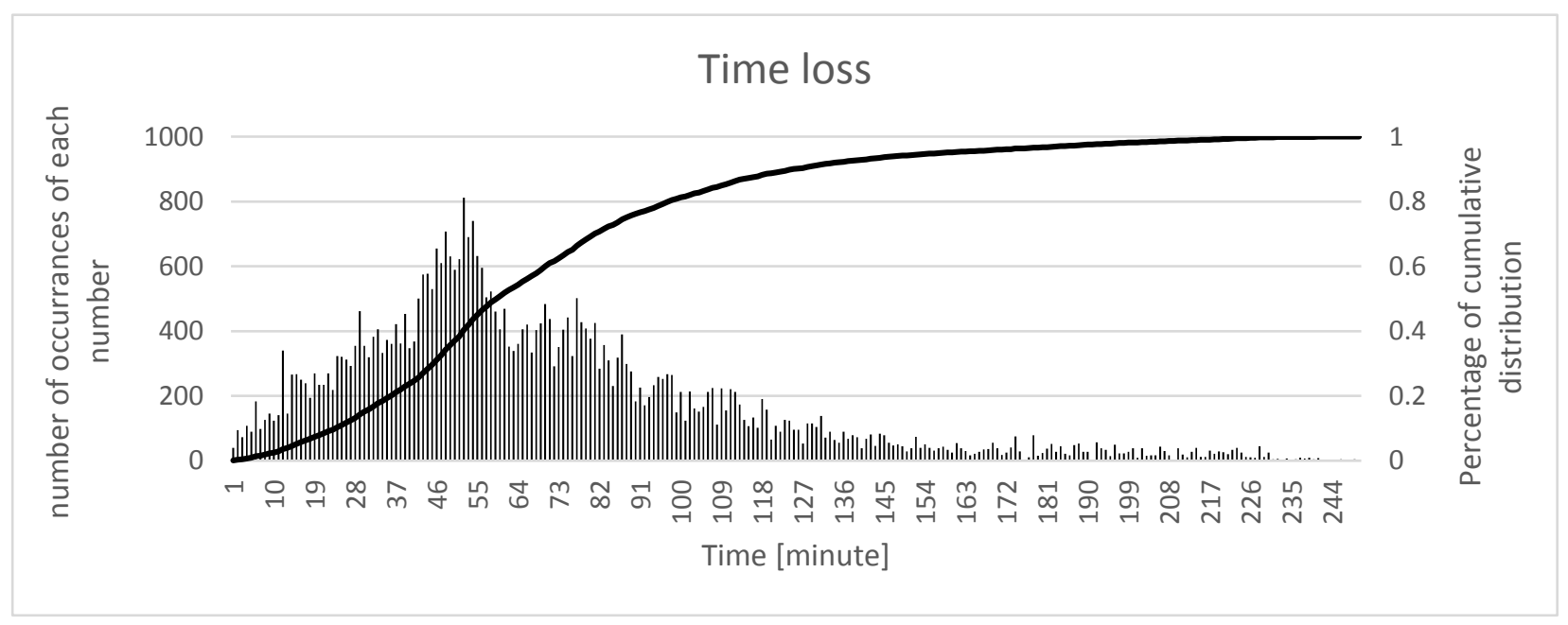

Figure 9 - frequency distribution of lost time due to stopover an QCS for charging 

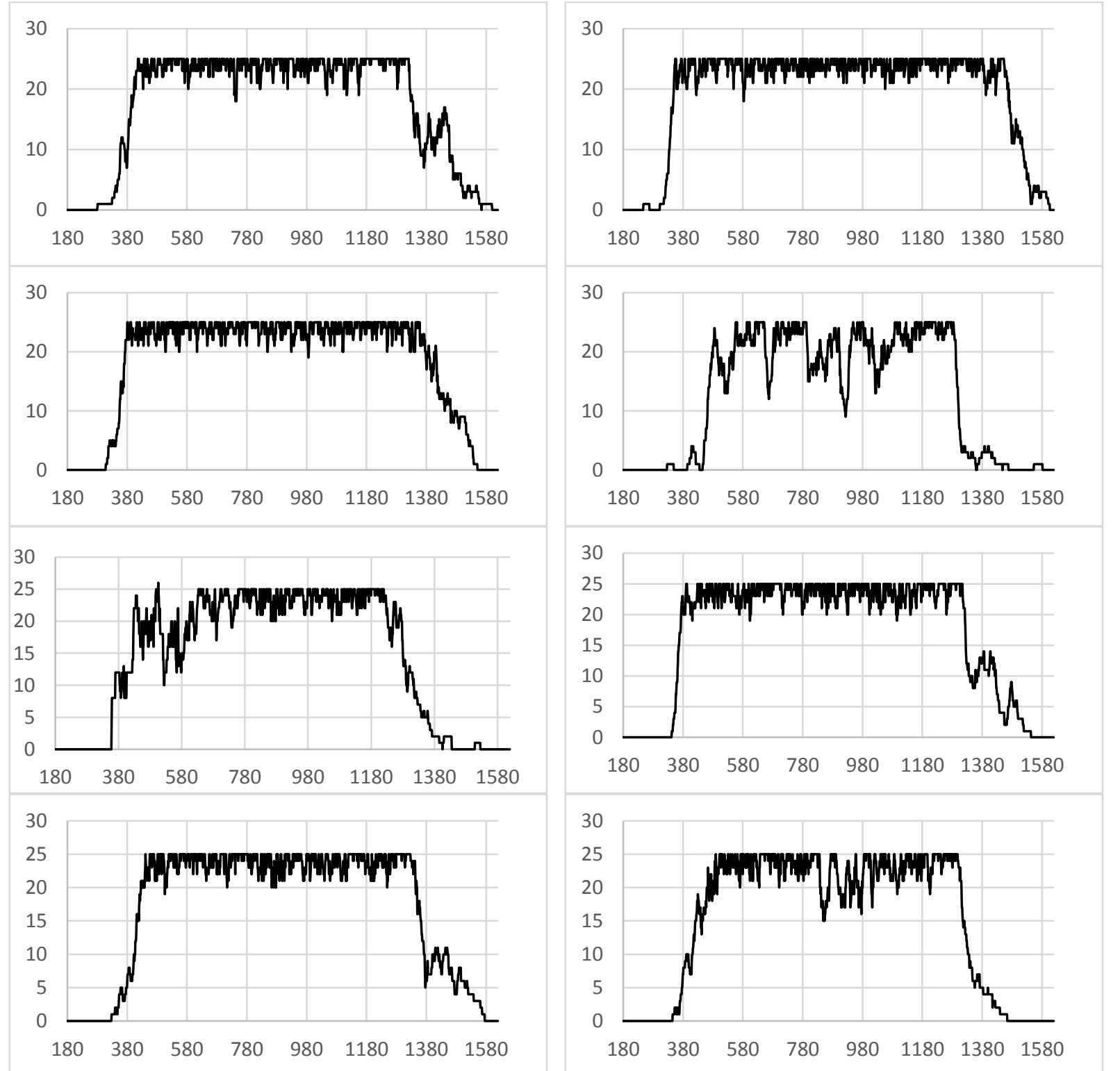

Figure 10 - Use of quick charging stations over the day. Horizontal axis represents time of the day from 180 to 1620 minutes and vertical axis represents the number of occupied chargers during the day

of the next day. From figure it is apparently visible that charging stations are completely occupied mostly during the morning and evening travel peaks.

Average time for planning a charging strategy for one vehicle took 190 milliseconds on a machine with following specifications. Processor type: Intel Xeon X5670, Number of processors 2, Cores/processor 12, Threads/processor 24, Clock frequency $2.95 \mathrm{GHz}$, Memory $128 \mathrm{~GB}$.

\section{Conclusion and future work}

A planning simulation model is presented which evaluates the feasibility of electric vehicles driving range when recharging is considered at home, work or at quick charging station. The daily schedules predicted by FEATHERS for whole population of Flanders, Belgium are passed to the charging planner that finds the optimal charging location for users who cannot complete their planned schedule without an intermediate charging. A new charging activity is added in the schedule after assigning location to specified trip. The total duration of the schedule is adjusted due to insertion of charging activities. All the chargers are installed around Brussels, which makes 
difficult for BEV users from farfetched areas of Flanders to reach and recharge at these stations. It is clear from the results that different quick charging stations attract different number of customers due to their reachability. The results have also shown that average travel time is greater than average charging duration, which is a major prevention to adoption of this charging behavior. This travel time could be minimized by better location optimization of quick chargers. Our results can serve as a good start for future study in designing better constraints. This leads to more accurate estimate on the battery consumption and recharging, time so that it would give a more accurate recharging planning. It can support facility location decisions as well to be compared with existing work. A similar kind of technique may be employed to deal with more difficult problem such as the location routing problem for an EV fleet.

The presented framework has the ability to be extended so that it could be helpful to find the candidate positions for installation of new infrastructure. For future research, we may proceed the following studies: (1) a travel execution model which takes care of more accurate street address based travel time and re-optimizes the booked charging requests (2) the investigation of the topics of the optimal deployment for new charging stations.

\section{References}

[1] J. Van Mierlo, G. Maggetto, and P. Lataire, "Which energy source for road transport in the future? A comparison of battery, hybrid and fuel cell vehicles," 12th Int. Conf. Emerg. Nucl. Energy Syst., vol. 47, no. 17, pp. 2748-2760, Oct. 2006.

[2] S. Amjad, S. Neelakrishnan, and R. Rudramoorthy, "Review of design considerations and technological challenges for successful development and deployment of plug-in hybrid electric vehicles," Renew. Sustain. Energy Rev., vol. 14, no. 3, pp. 1104-1110, Apr. 2010.

[3] D. Chandran and M. Joshi, "Electric vehicles and driving range extension-A Literature review," J. Adv. Veh. Eng., vol. 2, no. 4, 2016.

[4] J. Dong, C. Liu, and Z. Lin, "Charging infrastructure planning for promoting battery electric vehicles: An activity-based approach using multiday travel data," Transp. Res. Part C Emerg. Technol., vol. 38, pp. 44-55, Jan. 2014.

[5] H. Qin and W. Zhang, "Charging scheduling with minimal waiting in a network of electric vehicles and charging stations," presented at the Proceedings of the Eighth ACM international workshop on Vehicular inter-networking, 2011, pp. 51-60.

[6] F. Zhu, Y. Yao, W. Tang, and D. Chen, "A high performance framework for modeling and simulation of large-scale complex systems," Spec. Sect. Note New Trends Data-Aware Sched. Resour. Provisioning Mod. HPC Syst., vol. 51, pp. 132-141, Oct. 2015.

[7] I. Hussain et al., "Organizational-based model and agent-based simulation for long-term carpooling," Future Gener. Comput. Syst., vol. 64, pp. 125-139, Nov. 2016.

[8] C. Ma, J. Rautiainen, D. Dahlhaus, A. Lakshman, J.-C. Toebermann, and M. Braun, "Online Optimal Charging Strategy for Electric Vehicles," 9th Int. Renew. Energy Storage Conf. IRES 2015, vol. 73, pp. 173-181, Jun. 2015.

[9] Gonçalo Cardoso et al., "Stochastic Programming of Vehicle to Building Interactions with Uncertainty in PEVs Driving for a Medium Office Building," in 39th Annual Conference of the IEEE Industrial Electronics Society, 2013.

[10] M. Alonso, H. Amaris, G. J. Germain, and M. J. Galan, "Optimal Charging Scheduling of Electric Vehicles in Smart Grids by Heuristic Algorithms," Energies, vol. 7, no. 4, 2014.

[11] Y. He, B. Venkatesh, and L. Guan, "Optimal Scheduling for Charging and Discharging of Electric Vehicles," IEEE Trans. Smart Grid, vol. 3, no. 3, pp. 1095-1105, Sep. 2012.

[12] J. Barco, A. Guerra, L. Muñoz, and N. Quijano, "Optimal routing and scheduling of charge for electric vehicles: Case study," ArXiv Prepr. ArXiv13100145, 2013.

[13] C. Le Floch, F. di Meglio, and S. Moura, "Optimal charging of vehicle-to-grid fleets via pde aggregation techniques," presented at the 2015 American Control Conference (ACC), 2015, pp. 3285-3291.

[14] S. Acha, T. C. Green, and N. Shah, "Optimal charging strategies of electric vehicles in the UK power market," presented at the Innovative Smart Grid Technologies (ISGT), 2011 IEEE PES, 2011, pp. 1-8.

[15] R. L. G. Latimier, B. Multon, H. B. Ahmed, F. Baraer, and M. Acquitter, "Stochastic optimization of an Electric Vehicle Fleet Charging with Uncertain Photovoltaic Production," presented at the 2015 International Conference on Renewable Energy Research and Applications (ICRERA), 2015, pp. 721-726.

[16] T. Lan, J. Hu, G. Wu, S. You, L. Wang, and Q. Wu, "Optimal charge control of electric vehicles in electricity markets," presented at the The 4th International Workshop on Intelligent Information Management Systems and Technology, 2011.

[17] E. B. Iversen, J. M. Morales, and H. Madsen, "Optimal charging of an electric vehicle using a Markov decision process," Appl. Energy, vol. 123, pp. 1-12, 2014.

[18] A. Foley, B. Tyther, P. Calnan, and B. Ó. Gallachóir, "Impacts of electric vehicle charging under electricity market operations," Appl. Energy, vol. 101, pp. 93-102, 2013.

[19] V.-L. Nguyen, T. Tran-Quoc, S. Bacha, and N.-A. Luu, "Charging strategies to minimize the energy cost for an electric vehicle fleet,” presented at the IEEE PES Innovative Smart Grid Technologies, Europe, 2014, pp. 1-7. 
[20] M. G. Vayá and G. Andersson, "Optimal bidding strategy of a plug-in electric vehicle aggregator in day-ahead electricity markets under uncertainty," IEEE Trans. Power Syst., vol. 30, no. 5, pp. 2375-2385, 2015.

[21] I.-L. Wang, Y. Wang, and P.-C. Lin, "Optimal recharging strategies for electric vehicle fleets with duration constraints," Transp. Res. Part C Emerg. Technol., vol. 69, pp. 242-254, Aug. 2016.

[22] A. Y. Lam, Y.-W. Leung, and X. Chu, "Electric vehicle charging station placement: Formulation, complexity, and solutions," IEEE Trans. Smart Grid, vol. 5, no. 6, pp. 2846-2856, 2014.

[23] Y.-W. Wang, "An optimal location choice model for recreation-oriented scooter recharge stations," Transp. Res. Part Transp. Environ., vol. 12, no. 3, pp. 231-237, May 2007.

[24] Y.-W. Wang, "Locating battery exchange stations to serve tourism transport: A note," Transp. Res. Part Transp. Environ., vol. 13, no. 3, pp. 193-197, May 2008.

[25] Y.-W. Wang and C.-C. Lin, "Locating road-vehicle refueling stations," Transp. Res. Part E Logist. Transp. Rev., vol. 45, no. 5, pp. 821-829, Sep. 2009.

[26] Y.-W. Wang and C.-R. Wang, "Locating passenger vehicle refueling stations," Transp. Res. Part E Logist. Transp. Rev., vol. 46, no. 5, pp. 791-801, Sep. 2010.

[27] S. A. MirHassani and R. Ebrazi, "A Flexible Reformulation of the Refueling Station Location Problem," Transp. Sci., vol. 47, no. 4, pp. 617-628, Sep. 2012.

[28] M. Kuby and S. Lim, "The flow-refueling location problem for alternative-fuel vehicles," Socioecon. Plann. Sci., vol. 39, no. 2, pp. 125-145, Jun. 2005.

[29] M. Hosseini and S. A. MirHassani, "Refueling-station location problem under uncertainty," Transp. Res. Part E Logist. Transp. Rev., vol. 84, pp. 101-116, Dec. 2015.

[30] M. Hosseini and S. A. MirHassani, "Selecting optimal location for electric recharging stations with queue," KSCE J. Civ. Eng., vol. 19, no. 7, pp. 2271-2280, 2015.

[31] T. Bellemans, B. Kochan, D. Janssens, G. Wets, T. Arentze, and H. Timmermans, "Implementation Framework and Development Trajectory of FEATHERS Activity-Based Simulation Platform," Transp. Res. Rec. J. Transp. Res. Board, no. Volume 2175 / 2010 Travel Forecasting 2010, Vol. 1, pp. 111-119, Dec. 2010.

[32] A. Perujo and B. Ciuffo, "The introduction of electric vehicles in the private fleet: Potential impact on the electric supply system and on the environment. A case study for the Province of Milan, Italy," Energy Policy, vol. 38, no. 8, pp. 45494561, Aug. 2010.

[33] L. Knapen, B. Kochan, T. Bellemans, D. Janssens, and G. Wets, "Activity based models for countrywide electric vehicle power demand calculation," in IEEE SmartGridComm2011 Workshop.

[34] M. Usman, L. Knapen, A. Yasar, T. Bellemans, D. Janssens, and G. Wets, "A framework for electric vehicle charging strategy optimization tested for travel demand generated by an activity-based model," presented at the The 3rd International Conference on Connected Vehicles \& Expo (ICCVE 2014), 2014.

[35] M. Usman et al., "A coordinated Framework for Optimized Charging of EV Fleet in Smart Grid," 11th Int. Conf. Future Netw. Commun. FNC 2016 13th Int. Conf. Mob. Syst. Pervasive Comput. MobiSPC 2016 Affil. Workshop, vol. 94, pp. 332339, 2016.

[36] F. Nemry, G. Leduc, and A. Muñoz, "Plug-in Hybrid and Battery-Electric Vehicles: State of the research and development and comparative analysis of energy and cost efficiency," Institute for Prospective and Technological Studies, Joint Research Centre, 2009.

[37] Federal Planning Bureau, "Transportdatabanken : Indicator PARC010 428," 2009. [Online]. Available: http://www.plan.be.

[38] Eco-movement, "Eco-movement." [Online]. Available: www.oplaadpalen.nl. 